\title{
Reattach to Rebond; Restorative Management of a subgingival crown fracture
}

\section{Vivek. A ${ }^{1}$, M.C. Ponappa ${ }^{2}$, K.C. Ponnappa ${ }^{3}$, Neha. $S^{4}$, Nanjamma K.K}

1. Post graduate student, Dept Of Conservative Dentistry \& Endodontics, Coorg Institute Of Dental Sciences, Virajpet

2. Professor, Dept OfConservative Dentistry \& Endodontics, Coorg Institute OfDental Sciences, Virajpet

3. Professor \& Head of Department, Dept Of Conservative Dentistry \& Endodontics, Coorg Institute Of Dental Sciences, Virajpet

4. Senior lecturer, Dept OfConservative Dentistry \& Endodontics, Coorg Institute OfDental Sciences, Virajpet

5. Reader, Dept OfConservative Dentistry \& Endodontics, Coorg Institute OfDental Sciences, Virajpet

Corresponding author:

\section{DrVivek A}

Post graduate student,

Dept of Conservative Dentistry \& Endodontics,

Coorg Institute Of Dental Sciences,

Virajpet

Phone: 8891919996, 8136996886

Email - vivekintemail@gmail.com

\begin{abstract}
Long term survival of an endodontically treated tooth is always challenging as it is more prone to fracture when the full coverage restoration is delayed or is not at all given. Complicated crown and root fracture of such teeth is a cause of post endodontic failure. Re-restoring such teeth is bothersome as it depends on the location and extend of fracture line, affected tooth, and its relationship with the gingiva and alveolar crest. The following case report describes the reattachment of a fractured fragment of an endodontically treated maxillary right first premolar using flowable composite, after reinforcement with a fibre post, followed by fabrication of metal ceramic crown.
\end{abstract}

Key words - endodontically treated teeth, fracture re attachment, sub-gingival fracture, fibre-reinforced post, supra gingival finish line. 


\section{Introduction}

Endodontic procedures include mechanical as well as chemical events that can interfere with the natural stress-strain distribution in the tooth structure, which can collectively risk the fracture resistance of the tooth. So, rehabilitating an endodontically treated tooth having a complicated crown or crown root fracture is always a challenge to the dental practitioner and thus a common cause for post endodontic failure. ${ }^{1}$

For a post endodontic fracture, the mode of treatment depends on the affected tooth, type, location, extent of the fracture and its relationship with the gingiva and alveolar crest. ${ }^{2}$ Clinical management of such fractures may vary from simple reattachment to full crown with or without allied procedures, and if given to a hopeless prognosis, extraction has to be performed.

The following case report describes the reattachment of a fractured fragment of an endodontically treated maxillary right first premolar using flowable composite, after reinforcement with a fiber post, followed by fabrication of metal ceramic crown.

\section{Case Report}

A 26 year old female patient reported to the department of Conservative dentistry and

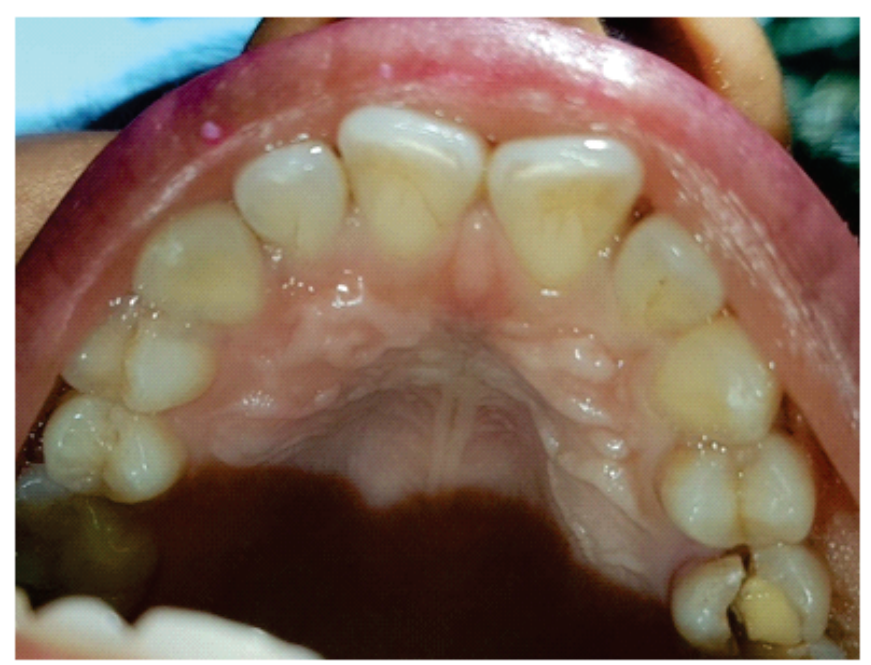

FIG. 1 - FRACTURED UPPER SECOND PREMOLAR
Endodontics with a fractured maxillary right first premolar, which was root canal treated but post endodontic restoration with a full coverage crown was not performed. (Figure 1)

Hard and soft tissue examination revealed a mobile palatal fragment, which was barely attached to rest of the tooth gingivally along with Class II resin composite restoration sealing the pulp chamber.

Radiograph revealed a satisfactory root canal obturation. Since there was no tooth structure loss, re-positioning of the fractured fragment in close approximation to the tooth remnant was planned.

The patient was explained regarding the procedures involved namely, surgical crown lengthening followed by the reinforcement of the tooth with a fiber post, reattachment of the fractured fragment and final restoration with a metal ceramic crown.

\section{Clinical Procedure}

There was an oblique fracture, involving the palatal half of the crown, with the fracture line extending from enamel, dentin, and to the cementum.

The fractured tooth fragment was gently removed under local anaesthesia (Figure 2) and was stored in normal saline.

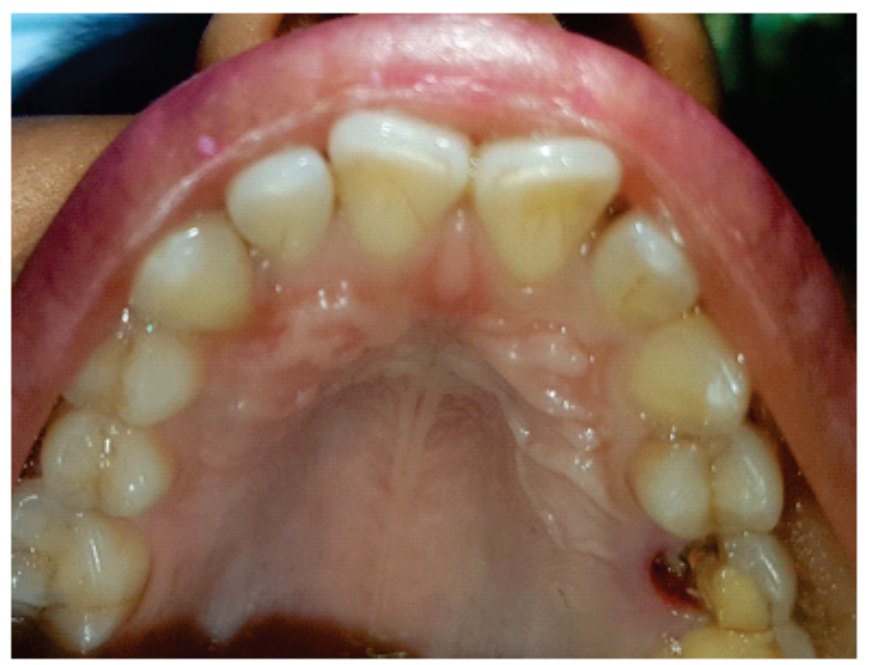

FIG. 2 - AFTER REMOVAL OF FRACTURED FRAGMENT 


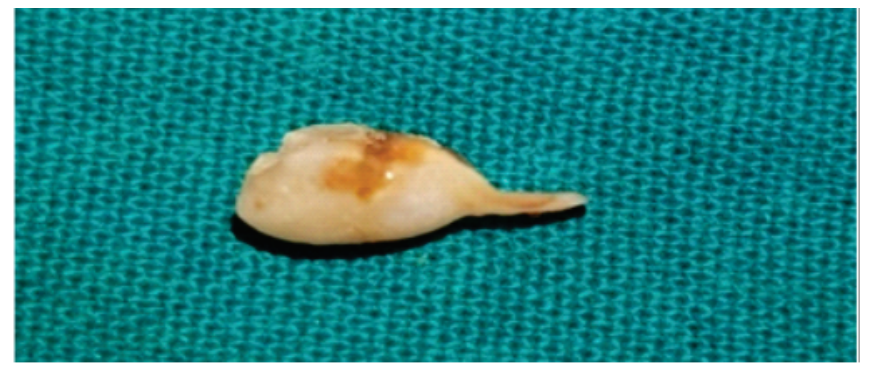

FIG. 3A - FRACTURED FRAGMENT

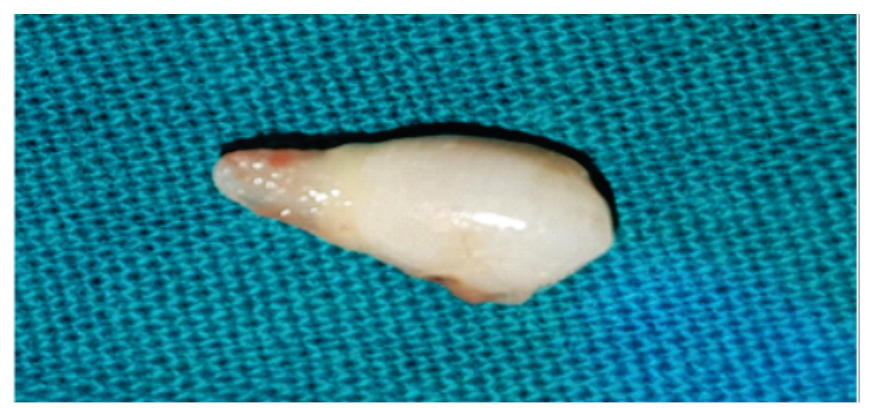

FIG. 3B - FRACTURED FRAGMENT

Following the principles of biological width, palatal gingivectomy was performed to expose the tooth structure around the fracture line, which was fading out subgingivally.

The existing class II composite restoration was removed, followed by preparation of post space with peeso reamer. A pre-fabricated fibre post of appropriate size was selected and was cemented using the dual cure resin cement (Rely XARC, 3M).

A channel preparation was done on the fractured fragment in order to accommodate the post and new composite access restoration. (fig 5).

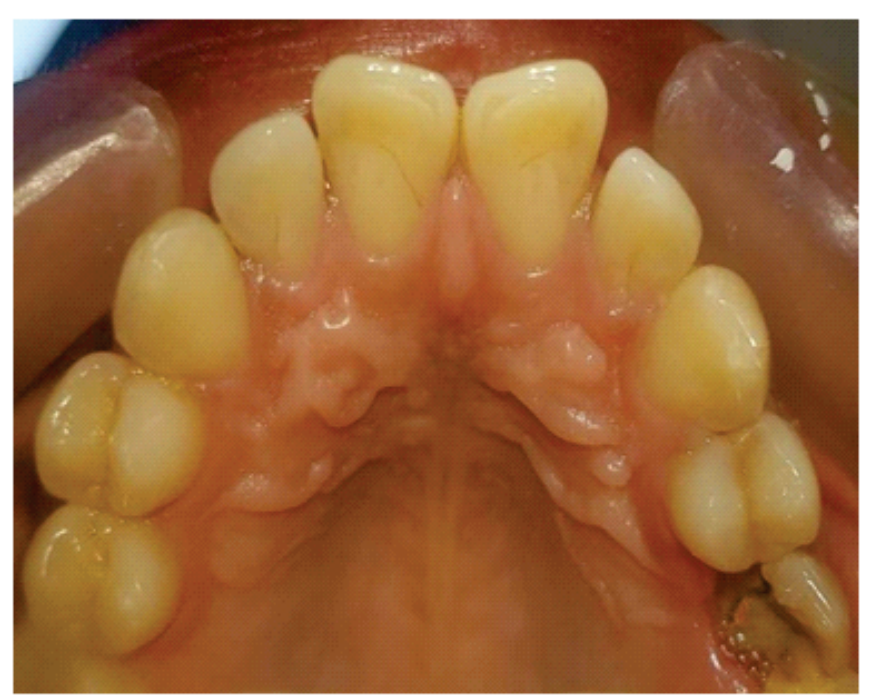

FIG. 4 - AFTER CROWN LENGTHENING

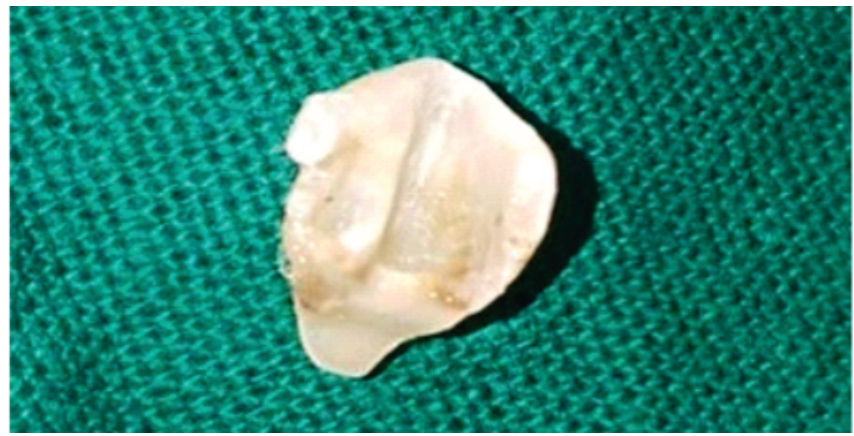

FIG. 5 - CHANNEL PREPARATION ON THE FRAGMENT

After maintaining a dry field and confirming the approximation of the fragment to the fracture line, without any further mechanical preparation, the surface of the tooth remnant was acid etched and bonded.

The fractured fragment was surface treated with etchant and bonding agent extra orally, followed by its reattachment using flowable composite (tetric flow, ivoclar).

In the same appointment, following shade selection, the tooth preparation was carried out with the reduction for a PFM crown, with equigingival shoulder margin in the buccal aspect and supra gingival shoulder margin on the palatal aspect.

Impression of the prepared tooth was obtained with addition silicon based impression material (EXPRESSTM,3M EPSE) using putty wash impression technique.

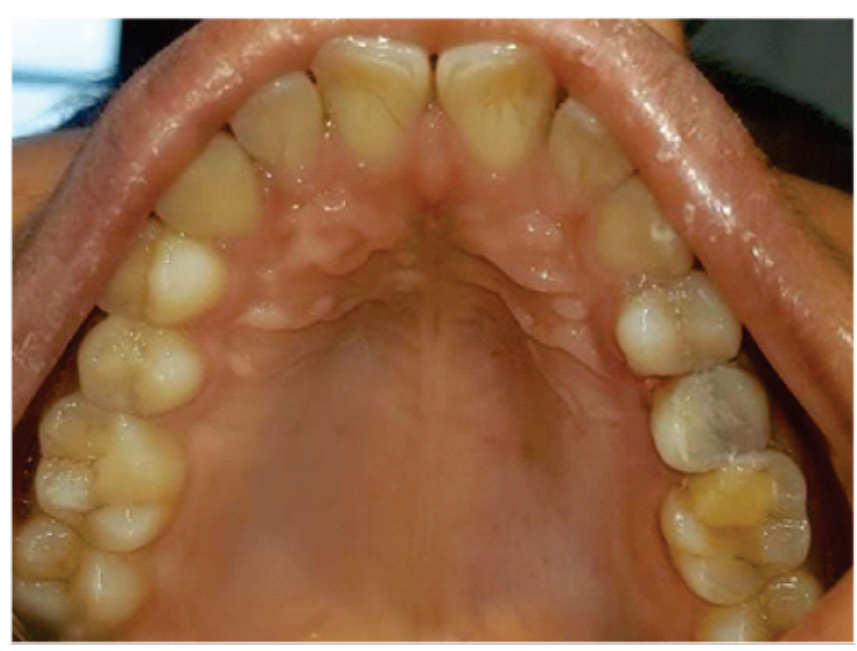

FIG. 6 - REATTACHED FRAGMENT AFTER INSERTION OF FIBRE POST AND CORE BUILDUP WITH COMPOSITE 


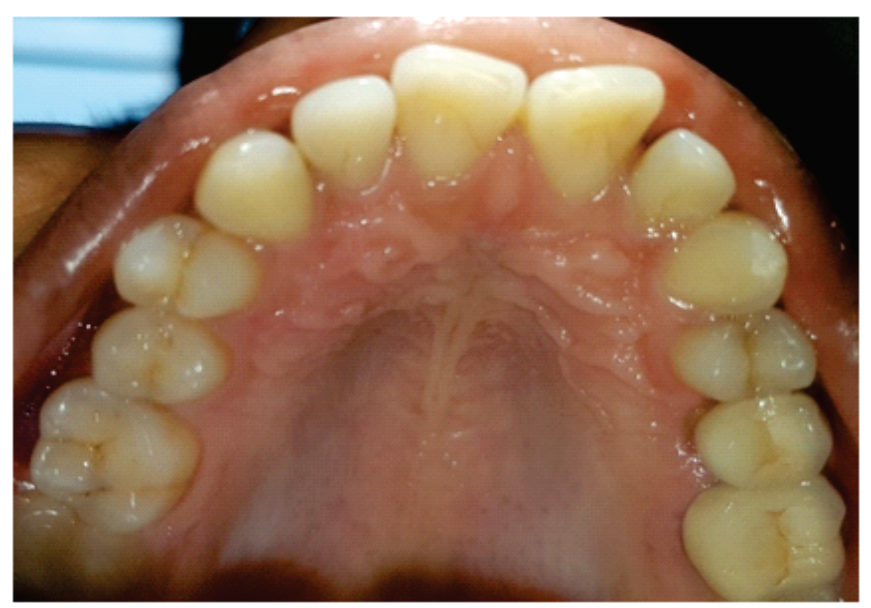

FIG. 7 - FINAL RESTORATION WITH CERAMIC CROWN

Temporisation was done with resin based temporary crown material (Revotek) and patient was recalled after one week.

In the subsequent appointment, fabricated metal ceramic crown was assessed for fit, and was cemented using glass ionomer based luting cement.

\section{Discussion}

The fracture resistance of the endodontically treated teeth weaken due to the loss of the tooth structure and this has greater importance than any possible changes affecting the proportion of collagen or the moisture content. A large epidemiologic survey reported that the long-term functional survival of endodontically treated permanent teeth was $97.1 \%$ after 8 years, however, the major reasons behind the failure of such endodontically treated teeth were coronal and/or radicular tooth fractures. ${ }^{3}$

A complicated crown or crown root fracture of an endodontically treated tooth is always bothersome as it requires profound clinical skills in endodontics, periodontics, and restorative dentistry. ${ }^{3}$ In this clinical scenario, an oblique fracture of endodontically treated upper right first premolar involving the enamel, dentin, and cementum of the palatal half of the crown with an extension of fracture line $2 \mathrm{~mm}$ to the root surface was observed.

There was no significant tooth structure loss, so the re-positioning of the fractured fragment in close approximation was possible. Advances in dental bonding technology have enabled to achieve excellent results in reattachment procedure provided that the biologic factors, materials, and techniques are logically assessed and managed. 4 The treatment plan has been made after a thorough evaluation of the periodontal, endodontic, coronal and occlusal status.

Other factors also might influence the choice of technique, such as the extension of fracture, quality of fit between fragments and the fracture pattern. The fractured fragment was stored in saline to prevent dehydration and collapse of the dentinal collagen. ${ }^{5}$ Many of the 5th generation bonding agents tend to increase the fracture resistance of reattached coronal fragments when used in conjunction with unfilled resin. ${ }^{4}$

Fragment re-attachment in an anterior tooth assure better and long lasting aesthetics than any tooth-colored restoration, as it displays optical properties similar to rest of the tooth and also restores its original shape and contour. It is biologically more acceptable as it would contact the soft tissues to which it adapts well by its smooth enamel surface. Also, it elicits a positive psychosocial response for the preservation of the natural tooth structure. ${ }^{6}$

Extensive damage to the tooth structure along with missing fragment justifies reinforcement using fiber posts. There are several advantages in using tooth colored fiber posts. It is more aesthetic, and it easily bonds to tooth tissue, with a modulus of elasticity similar to that of dentin, with least chances of fracture.

In earlier days, fracture reattachment procedure was performed using cast metal post and core [Chosack and Eildeman, 1964]. The newer varieties of non-metallic posts are available, which are made of either fiber or ceramic reinforced materials like carbon, quartz or glass in an epoxy matrix. ${ }^{4} \mathrm{~A}$ combination of glass fiber post with composite core, along with recent advances in adhesive techniques and materials, one can create a Monobloc, which is a 
multi-layered structure with no inherent weak interfaces. The uniqueness of this system is that it reinforces the tooth structure.

Therefore, the integrity of the final endodonticrestorative continuum monobloc approaches that of the original healthy tooth itself. An add on benefit of using fiber posts is that it helps in stress equal stress distribution to remaining radicular dentin.7 It is being reported that the supragingival margin is the best biological place for a restoration.

Supragingival margins stay away from the periodontal tissues, and thus have the following advantages:

- Preservation of tooth structure during tooth preparation.

- Impressions are more predictable, with minimal or no cord packing.

- Provisional restorations are easier to make, and the soft tissues will be healthier when the patient returns for cementation of the final restoration.

Excess cement can be removed when the margin is visible. $8 \mathrm{But}$, when the fracture site is subgingival or intraosseous, orthodontic extrusion along with a post retained crown may be required [Barteiri et al, 1990]. However, in cases with minimal biologic width invasion, the individual can restore the biologic width by itself provided assiduous plaque control is done.

The prognosis of reattached teeth also depends on the contour and surface finishing of the subgingival restoration. If the invasion of biologic width is minimal, satisfactory aesthetics and function can be achieved, without conventional flap surgery, however requiring long term follow up.

\section{Conclusion}

Reattachment of the intact fractured fragment can be considered as a conservative approach for maintaining aesthetics and function.

This procedure helps us to preserve natural tooth structure at its maximum. Many other aspects dominate the choice of technique or even the type of materials used for fragment reattachment. The major priority is of patient cooperation and also understanding the limitations of the treatment which is of utmost importance for a good prognosis. Reattachment proved to be a successful technique in this case for restoring aesthetics and function.

\section{References}

1. D. Barsness, B. and Harris Roach, S. Restoration Of Root Canal-Treated Teeth, an adhesive dentistry perspective. 1st ed.: 2018 Springer International $\mathrm{Pu}$.

2. Cohen. S, Burns. R.C.; ed. by Trope. M, Chivian. N, Sigurdsson. A.: Pathways of the pulp, 8 th edn., Mosby, 603 - 649.2002.

3. Kemal Çalışkan, M. and Tolga Ceyhanlı, K. Reattachment of endodontically treated lateral incisor with supragingivally complicated crown fracture using fiber-reinforced post. Dental Traumatology, 2011,27(4), pp.305-308.

4. Divakar HD, Nayak MO, Shetty RA. Changing concepts in fracture reattachment of teeth-A case series. Endodontology. 2007; 2:27-35.

5. Chu.F.C, Yim. T.M, Wei. S.H.; Clinical consideration for reattachment of tooth fragment. Quint. Int. 2000: 3 1(6), 385 -391.

6. Vivekananda Pai, A. and Kamath, M. Reattachment and composite crowning of a fractured, endodontically treated tooth - a case report. Journal of Conservative Dentistry, 2005, 8(1), p.19.

7. Tay FR, Pashley DH. Monoblocks in root canals: A hypothetical or tangible goal. J Endod 2007; 33:391-8.

8 Shenoy, A., Babannavar, R. and Shenoy, N. Periodontal considerations determining the design and location of margins in restorative dentistry. Journal of Interdisciplinary Dentistry, 2012,2(1), p.3. 